\title{
Impact of liquid fuel on the flame response at elevated pressure
}

\author{
Virginel Bodoc ${ }^{\star 1}$, Julien Garraud ${ }^{1}$, Pierre Gajan ${ }^{1}$ \\ ${ }^{1}$ DMPE, ONERA, Toulouse, France \\ *Corresponding author email : virginel.bodoc@onera.fr
}

\begin{abstract}
This paper details the experimental study performed at ONERA on the LACOM test bench. Thermoacoustic transfer functions of a full scale gas turbine injector operating up to 5 bars have been measured. The fuels correspond respectively to Jet A1 fuel and the Alcohol to Jet (ATJ) alternative fuel. The modulation of the airflow was done using a siren. Two microphones have been used to record the acoustic response of the system. The characterization of the flame response relies also on the chemiluminescence emissions of $\mathrm{OH}^{\star}$ radical formed by combustion
\end{abstract}

\section{Keywords}

Flame Transfer Function, chemiluminescence, Phase Doppler Anemometry.

\section{Introduction}

Combustion instability observed in jet engines results from the coupling between the acoustic pressure/velocity field and the unsteady heat released by the flame. This coupling induces an important amplification of the pressure and temperature oscillations amplitude that can lead to the physical destruction of the injection system and/or combustor. The physical factors affecting engine operability (e.g. combustion stability) are not yet clearly known. Two fuels were tested within this work in order to tackle potential fuel impact on fuel spray and combustion stability.

\section{Material and Methods}

The experimental setup represents an upgrade of the LOTAR setup and is installed on the LACOM test bench. With respect to LOTAR setup [1] [2], a variable section sonic throttle was installed downstream the combustion chamber in order to allow operating at pressures higher than the ambient pressure (Figure 1).

The main elements of the test section are represented by the water cooled test chamber and the plenum that are separated by the injection system. The upstream part corresponds to a plenum where both the air and the fuel lines are connected to. The downstream part is equipped with transparent windows allowing the optical access to the combustion region. In the frame of reference of Figure 1, the airflow is directed from left to right.

The injection system was defined during the TLC program and provided by Safran. It consists in a pilot zone on the axis, surrounded by two axial swirlers and a multipoint injection zone at the periphery ( 24 perforations), surrounded by a radial swirler.

The pulsating gaseous flow is obtained by a siren placed upstream of the test section. The operating principle is based on a sonic jet that is periodically chopped by a sprocket wheel. The rotational velocity of the sprocket wheel sets the pulsation frequency. The blocked area of the sonic nozzle, and consequently the airflow pulsation level, can be varied by changing the relative position between the nozzle and sprocket wheel axis.

For the characterization of the spray, a Phase Doppler Anemometer was used. The system provides the size and two components of the velocity of drops crossing the probe volume. The probe volume is the result of the intersection of two laser beams; it has an oval shape, 
$0.3 \mathrm{~mm}$ in diameter and about $4 \mathrm{~mm}$ in length. The system used is a PDA from Dantec, which works with two $300 \mathrm{~mW}$ lasers (532 nm and $561 \mathrm{~nm}$ ).

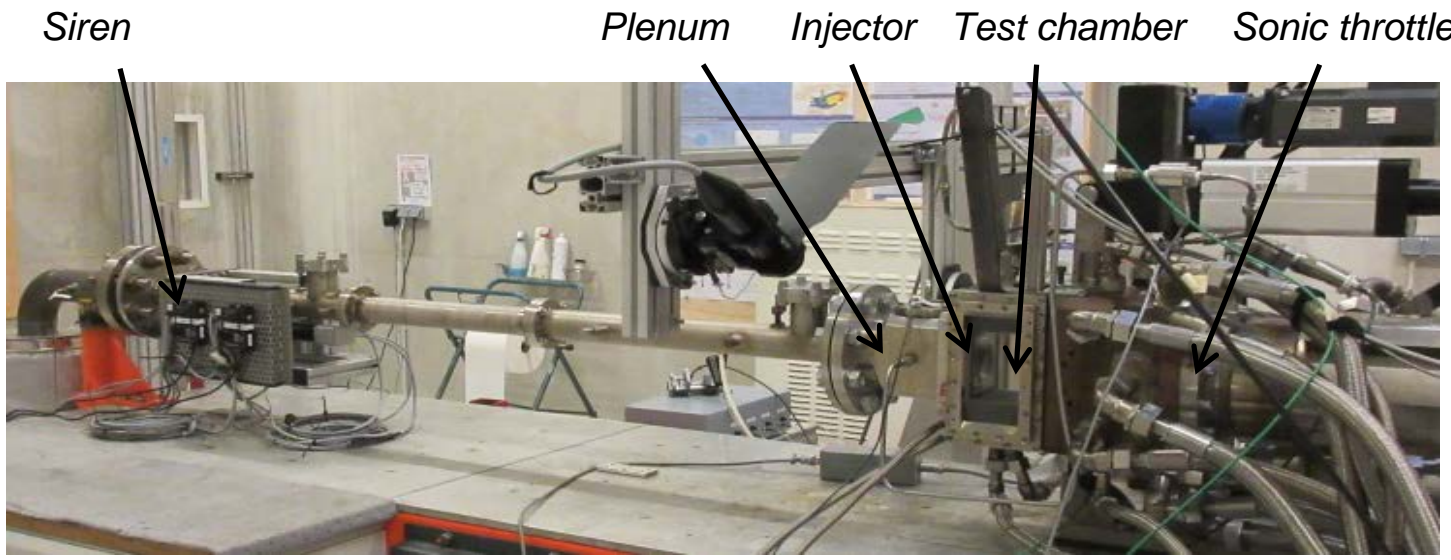

Figure 1. Test setup

The PDA optical setup is described in Figure 2. The green $(532 \mathrm{~nm}$ ) laser beams are located horizontally, in the same plane as the mean airflow and allow measuring the longitudinal component of the velocity vector. The yellow laser beams $(561 \mathrm{~nm})$ are aligned in a vertical plane and, when the corresponding measurement volume is placed along the OX axis, they provide the tangential component of the velocity. If the same measurement volume is placed along the vertical axis OY, the radial component of the velocity is measured. The identification of the last two components of the velocity is based on the hypothesis that the swirled flow is axisymmetric and the geometrical axis of the injector is identical to the aerodynamic axis of the swirling jet.

The measurements were taken at different longitudinal distances from the reference injection surface. For each run 50000 samples were recorded with a maximum recording interval of $20 \mathrm{~s}$. The validation rate (number of validated samples/total number of samples) was typically superior to $90 \%$.

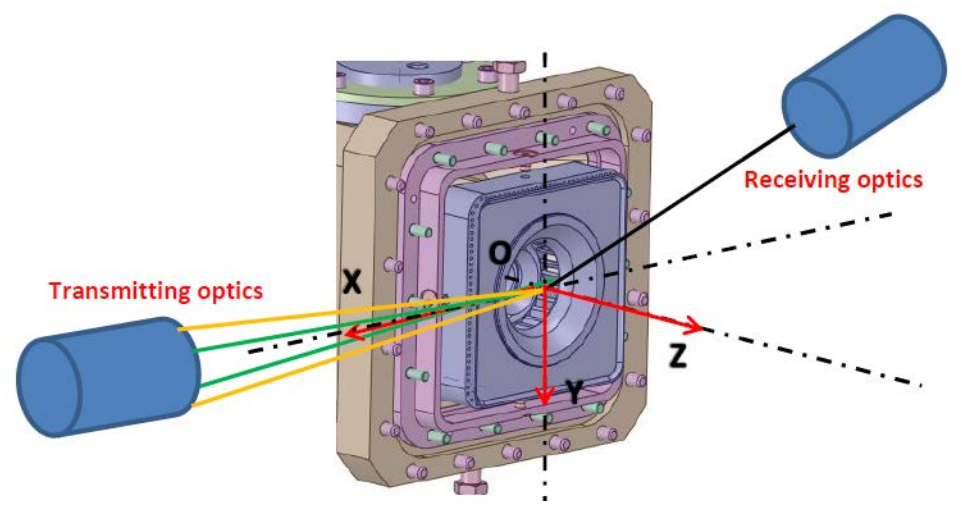

Figure 2. Sketch of the optical setup for the characterization of the fuel spray

For previous tests [1] performed at ambient pressure different methods were applied to analyse the flame response. One of them is based on the heat release fluctuations measurement from chemiluminescence intensity emitted by four different radicals $\left(\mathrm{OH}^{*}\right.$, $\left.\mathrm{CH}^{*}, \mathrm{C2}^{*}, \mathrm{CO}^{*}\right)$. Four PM tubes equipped with narrow-band interference filters recorded the integral $\mathrm{OH}^{*}, \mathrm{CH}^{*}, \mathrm{C2}^{*}, \mathrm{CO}^{*}$ flame emissions as a measure for the heat release $\mathrm{A}$ second one is based on the chemiluminescence of the unique $\mathrm{OH}^{*}$ radical. The two 
approaches were applied on the A1-fuel flame fueled only by the pilot zone. The main conclusion was that the tendencies obtained by both optical techniques are very similar. Consequently, within this work the analysis is limited to the determination of flame response only through the $\mathrm{OH}^{\star}$ emission.

By definition, the FTF represents the ratio of relative fluctuations of the heat release to the relative fluctuations of the velocity upstream the reactive zone and is a function of the frequency. It is mathematically expressed as:

$$
F T F(f)=\frac{\dot{Q}^{\prime} / \bar{Q}}{u^{\prime} / \bar{u}}
$$

where the fluctuations of velocity and heat release are normalized with their respective mean values [2].

Because the fluctuating components are complex functions of frequency, the FTF may be written as:

$$
F T F(f)=|F T F(f)| e^{i \cdot \varphi(\omega)}=\frac{\left|\dot{Q}^{\prime}(f)\right| \cdot \bar{u}}{\left|u^{\prime}(f)\right| \cdot \dot{\dot{Q}}} e^{i\left(\varphi_{\dot{Q}^{-}} \varphi_{u^{\prime}}\right)}
$$

The FTF is measured using a photomultiplier and a $\mathrm{OH}^{*}$ filter with the hypothesis that $\mathrm{OH}^{*}$ emission from the flame is directly proportional to the heat release [3]:

Figure 3 shows the setup used for the FTF instrumentation. The analysis of the acoustic field is based on unsteady pressure measurements obtained from microphones placed at two different locations upstream of the combustion chamber. Due to the high airflow temperature, the microphones were mounted on waveguides. Each acoustical probe (waveguide + microphone) was previously calibrated on a Kundt's tube. The characterization of the acoustic field is obtained by the two-microphones method developed by Abom [4]. This method is based on pressure signals measured simultaneously at two locations separated longitudinally from each other by a known distance. From this information the acoustic pressures and velocities may be calculated at different locations of the pipe section.

A photomultiplier (PM) tube equipped with a narrow-band interference filter centered at $308 \mathrm{~nm}$ records the integral $\mathrm{OH}^{*}$ flame emission through the access window as the measure for the heat release. Data acquisition and evaluation was performed with a Brüel \&Kjær Pulse system to obtain their respective auto-spectra and cross power spectrum density.

The FTF amplitude was evaluated as the ratio of normalized PM voltage fluctuation amplitude and normalized acoustic velocity amplitude.

$$
|F T F(\omega f)|=\frac{\left|I^{\prime}{ }_{O H}(f)\right| \cdot \bar{u}}{\left|u^{\prime}(f)\right| \cdot \bar{I}_{O H}}
$$

The FTF phase was evaluated as the phase difference between the PM and the acoustic velocity in position of microphone $2(\mu 2)$ in Figure 3 from the cross-power spectrum of the $\mathrm{PM}$ and microphone signals. 


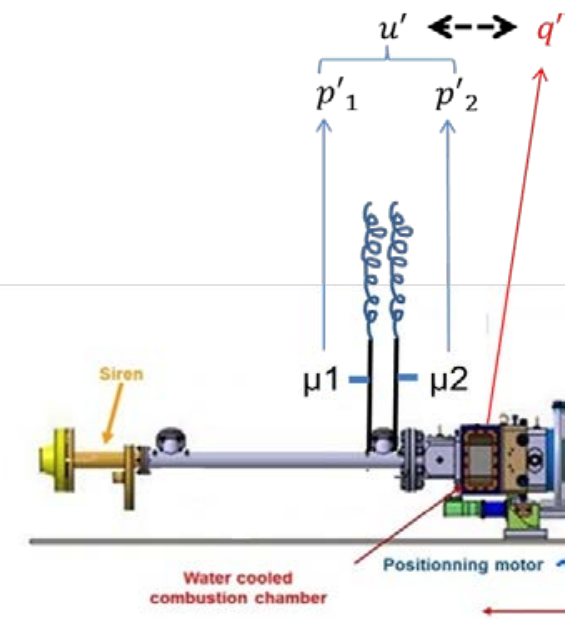

Figure 3: Experimental setup used for the flame description function measurement by an optical method

\section{Results and Discussion}

\section{Spray characterisation}

All the results obtained by PDA and presented hereafter were obtained for non-reactive conditions and for non-pulsating airflows. The operating pressure was of 5 bars and only the pilot circuit is fuelled.

Profiles of the mean longitudinal $\left(V_{z}\right)$, azimuthal $\left(V_{\theta}\right)$ and radial $\left(V_{r}\right)$ components of the velocity vector are plotted in Figure 4 for both fuels for $Z=20 \mathrm{~mm}$. As expected, the profiles of the velocity components clearly show that the flow topology behind the injection system corresponds to a swirling jet. The distribution of the longitudinal velocity across the test chamber section reveals that the flow is rather axisymmetric and the velocity maxima are displaced radially from the axis very close to the chamber walls.

The azimuthal velocity profile shows again the swirling behaviour of the dispersed flow. It can be observed that the azimuthal velocity value does not vanish at $R=0 \mathrm{~mm}$. It is assumed that, due to the swirling flow, the symmetry axis of the spray is not aligned with the symmetry axis of the injection system (OZ). The mean radial velocity is of the same order of magnitude when compared to longitudinal velocity component.

The profiles of fluctuating velocity components were also compared for ATJ (Alcohol to Jet alternative fuel) and JET-A1 sprays generated by the pilot nozzle (results non-presented here). The comparison of these profiles shows almost the same dynamic behaviour for drops of the two fuels.
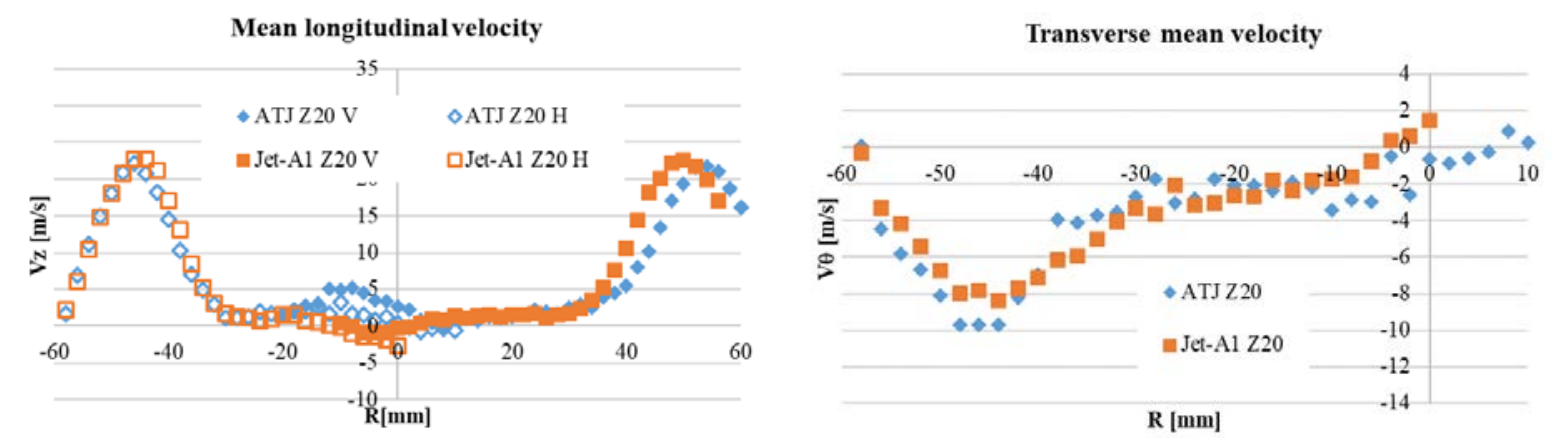


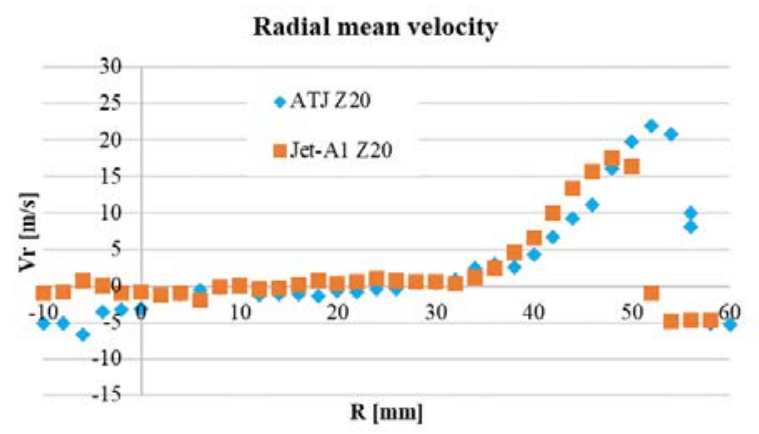

Figure 4. Comparison of mean velocity for Jet-A1 and ATJ fuels. Only the pilot line is fueled. $\mathrm{H}$ and $\mathrm{V}$ stand for the horizontal and vertical axis respectively

The D10 diameter profiles are compared in the next figure for different distances from the injection nozzle. Despite some scattering in the reported data, the main tendencies may be inferred. When only the pilot circuit is fuelled, the drops mean diameter is inferior to $18 \mu \mathrm{m}$, decreasing with the distance $Z$ to the injection plane and the distance $R$ to the injection axis. This behaviour is clearly due to the evaporation of the fuel drops and is proportional to the distance to the injection point in this high temperature environment.

The evolution of the ATJ droplets mean diameter with the distance to the injection section and the injection axis is plotted in Figure 5(left side graph). The right side graph in the same figure plots the droplet mean size measured for the Jet A1. Qualitatively, similar tendencies are obtained as in the case of the Jet-A1 fuel, i.e. a decrease of the drops mean size with the distance to the symmetry axis. However, the quantitative analysis of the two graphs reveals some differences. If these differences are relatively small (5-6\%) for $Z=20 \mathrm{~mm}$ on the symmetry axis, they increase up to $30 \%$ downstream in the flow and with the distance $\mathrm{R}$ to the symmetry axis.
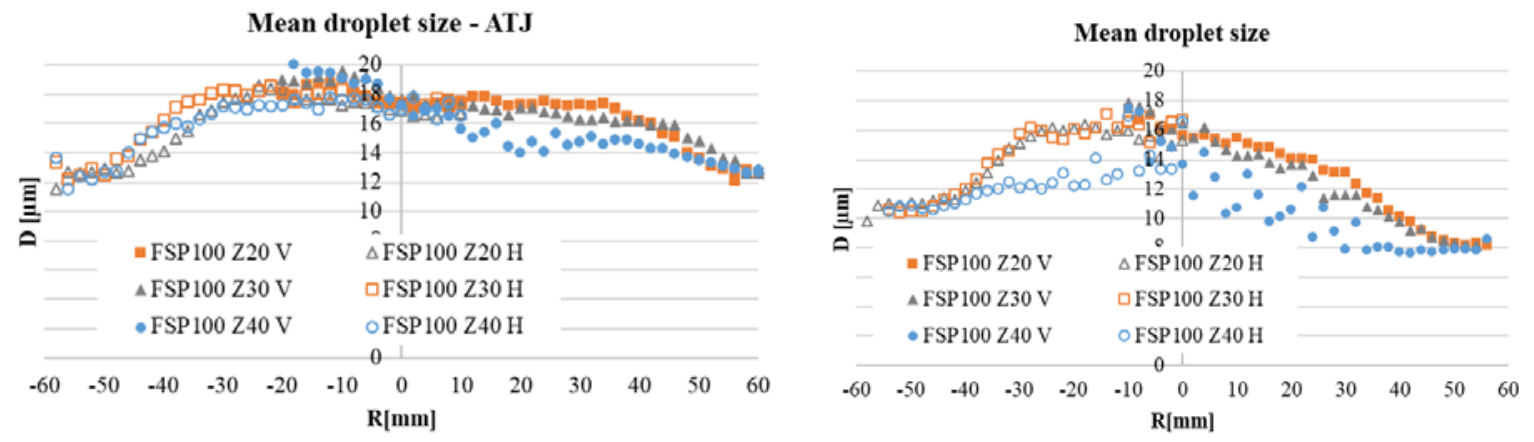

Figure 5. Drops mean size measured - Left: ATJ fuel, right: Jet-A1 fuel

\section{Flame response}

The flame response was measured with the optical method presented previously. Experiments were performed for one fuel injection configuration - only the pilot zone is fed for sake of simplicity.

The operating parameters for the evaluation of the flame transfer function (FTF) are listed inTable 1. Basically, the measurements were performed for three test conditions, one for the Jet-A1 and two for the ATJ. The objective was to evaluate both the influence of the pressure and that of the fuel on the FTF. For all tests, the air temperature and the global equivalence 
ratio (GER) were kept constant. In order to keep the same air bulk velocity through the injector, the air mass flow rate was varied for the two pressures. The fuel mass flow rate changed consequently.

Table 1 - Operating conditions for the flame transfer function measurement

\begin{tabular}{c|ccc}
\hline & Jet-A1 & ATJ & ATJ \\
\hline Pressure [bars] & 3 & 3 & 5 \\
Air temperature $[\mathrm{K}]$ & 500 & 500 & 500 \\
Air mass flow rate $[\mathrm{g} / \mathrm{s}]$ & 200 & 200 & 344 \\
Fuel mass flow rate $[\mathrm{g} / \mathrm{s}]$ & 6 & 6 & 10.5 \\
GER & 0.4575 & 0.4575 & 0.4575
\end{tabular}

For all the tests the pulsation frequency was varied from $10 \mathrm{~Hz}$ to $270 \mathrm{~Hz}$ for several acoustic velocity amplitudes. As a matter of fact, the velocity fluctuations at position II vary up to $8 \mathrm{~m} / \mathrm{s}$. However, the two mico-phones method used to compute the acoustic velocity from acoustic pressure measurements [4] is only valid for a frequency range that depends on the distance between the two microphones, the speed of sound and the Mach number. For the geometrical characteristics and the operating conditions used within this study, the application of this formula limits the use of the method for frequencies ranging between 175 $\mathrm{Hz}$ and $270 \mathrm{~Hz}$

The relationship between the $\mathrm{OH}^{\star}$ fluctuation amplitude and the frequency of the excitation is plotted for both fuels in Figure 6. All these values were obtained for the same position of the sprocket wheel with respect to the sonic throttle axis. This is equivalent to say that the blockage of the sonic throttle was kept constant through all these measurements. Three frequency peaks emerge at $20 \mathrm{~Hz}, 80 \mathrm{HZ}$ and $190 \mathrm{~Hz}$.It is observed that the flame response highly changes with the airflow modulation frequency except for a frequency range between $140 \mathrm{~Hz}$ and $220 \mathrm{~Hz}$ where the two curves overlap. The differences between the two plots are mainly dues to the different behaviors of the two fuels in reactive conditions.

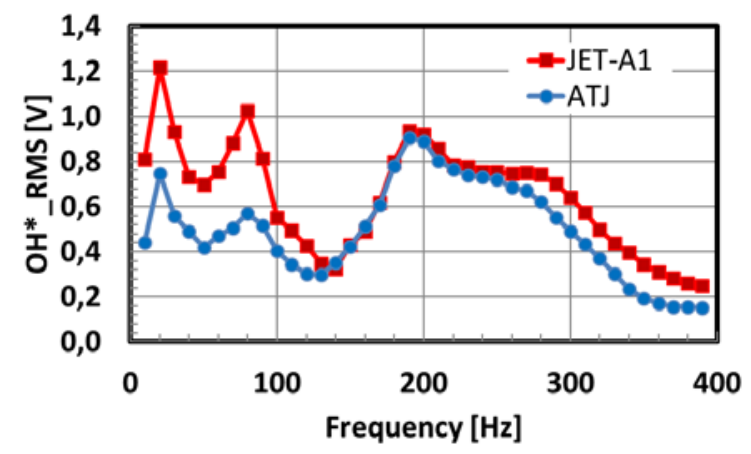

Figure 6. $\mathrm{OH}^{\star}$ emission fluctuation level obtained for $\mathrm{P}=3$ bars. For this test the sprocket wheel positions was kept constant for both tested fuels

The relationship between the characteristics of the excitation and the heat release rate is characterized by the Flame Transfer Function (FTF) between the upstream acoustic velocity and the unsteady heat release. The amplitude and phase relationships between the upstream excitation and the $\mathrm{OH}^{*}$ emission is plotted in Figure 7 for both fuels. An important 
influence of the excitation frequency on the amplitude of the FTF is observed. Moreover, the ATJ behavior exhibits higher FTF amplitude compared with the Jet-A1 one.

For the frequencies considered, the phase relationship between the upstream velocity fluctuation and the heat release rate is almost identical for both fuels. For both plots, the presence of two linear relationships may be observed for the investigated frequency range. This behavior corresponds to a constant delay between the two signals that can be calculated from the slope of these curves.

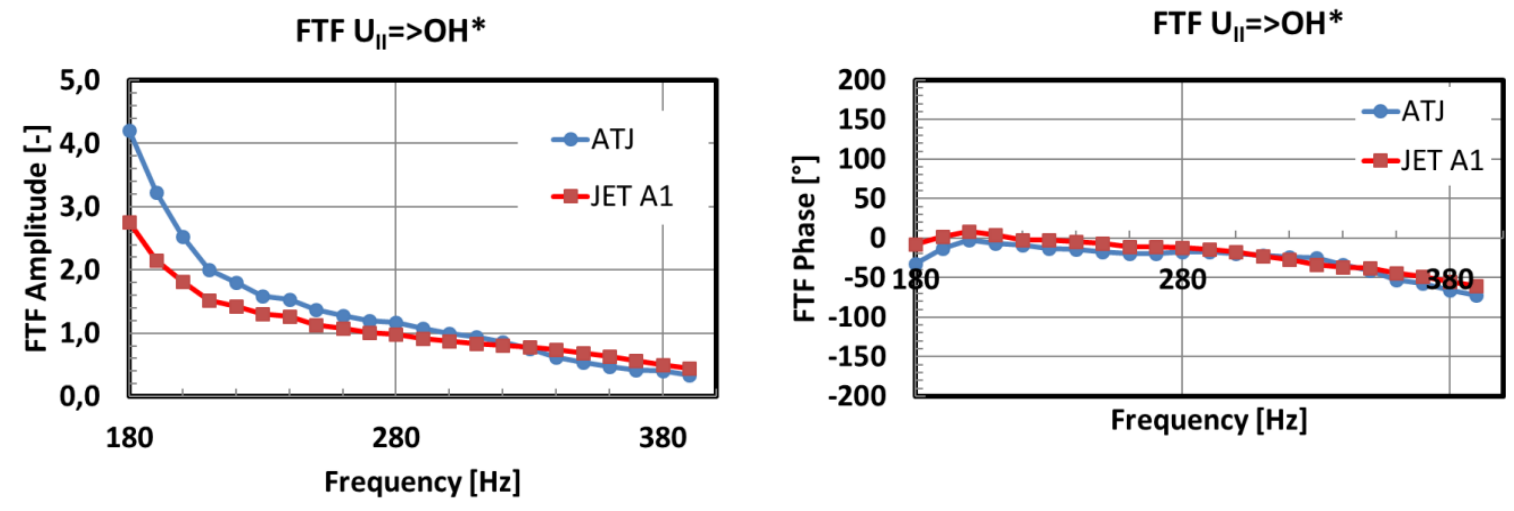

Figure 7. Flame Transfer Function obtained for a test chamber pressure of 3 bars. Influence of the fuel.

The respective influence of the frequency and the excitation level on the $\mathrm{OH}^{*}$ fluctuation at 5 bars are plotted in Figure 8. From the graphs the flame response highly changes with the airflow modulation amplitude but it seems to be independent of the excitation frequency, at least for small modulation amplitudes.
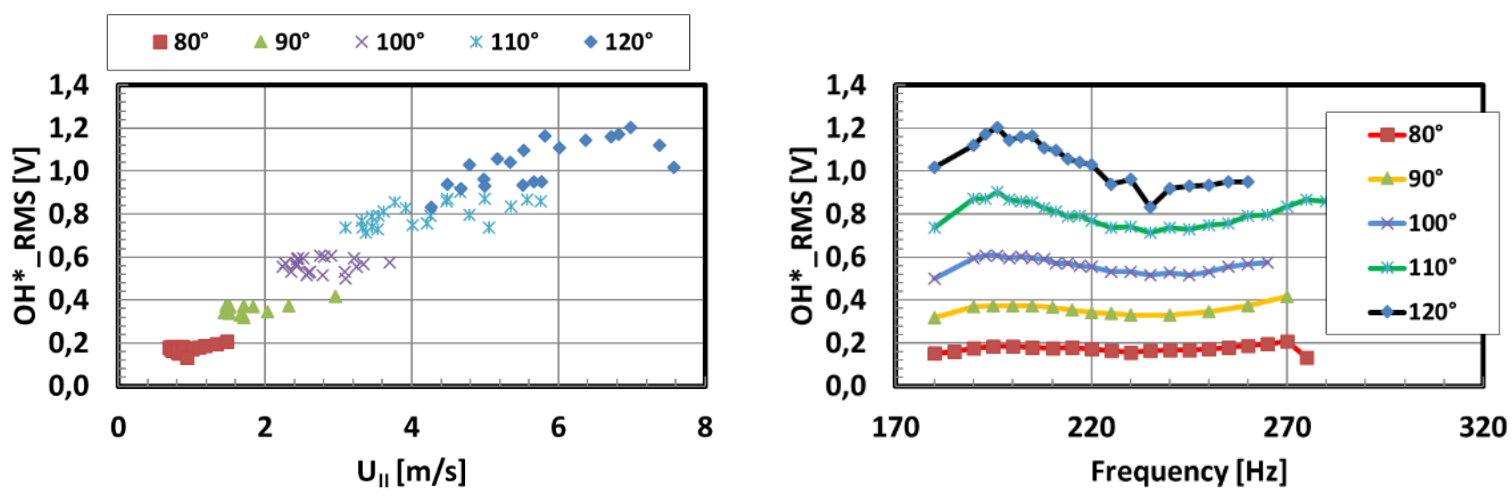

Figure 8. $\mathrm{OH}^{*}$ emission fluctuation level obtained for a pressure of 5 bars (ATJ fuel only). Left: evolution with the airflow pulsation amplitude, right: evolution with the pulsation frequency. The angular units correspond to the sprocket wheel positions

The influence of the excitation frequency and amplitude on the FTF is shown in Figure 9.The maximum of the FTF amplitude shifts to higher frequencies when the amplitude of the modulation increases. For frequencies above $200 \mathrm{~Hz}$, a weak influence of the pulsation amplitude on the amplitude of the FTF is thus observed. Concerning the phase relationship, the results are similar to those obtained at 3 bars. The linear behavior observed for frequencies above $220 \mathrm{~Hz}$ corresponds to a constant delay between the two signals which can be calculated from the slope of these curves. The results obtained indicate that this delay decreases with the amplitude of the excitation. 

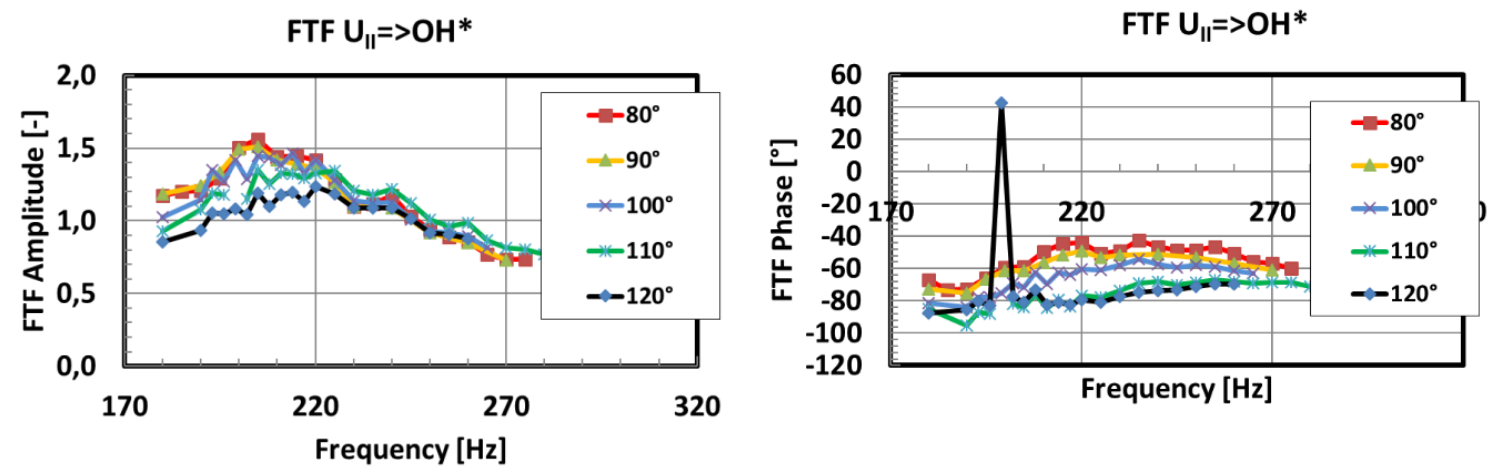

Figure 9. Flame Transfer Function obtained at a test chamber pressure of 5 bars. Influence of the acoustic velocity amplitude. The angular units correspond to the sprocket wheel positions

\section{Conclusions}

In this paper, an experimental study on the influence of two different fuels Jet-A1 and ATJ on the spray characteristics and on the flame response to acoustic excitations was performed under pressure conditions. For both fuels, the characterization of the spray was performed under similar conditions. The comparison of the drops velocity and size shows quantitative differences between Jet-A1 and ATJ from the point of view of both the atomization and evaporation processes.

Similarly, some differences between the two fuels were observed for the FTF for a pressure of 3 bars. As a matter of fact, for the investigated frequency range the difference in the FTF amplitude decreases with the frequency value.

The FTF was also measured for a flame fueled with ATJ fuel for a pressure of 5 bars. Tests performed for different pulsation frequencies and amplitudes show a sensitivity of the flame to the acoustic disturbance.

\section{Acknowledgments}

This work was performed within the European funded JETSCREEN project (grant agreement No 723525).

\section{References}

[1] A. Desclaux, PhD Thesis - Etude expérimentale du comportement linéaire et non linéaire d'une flamme diphasique soumise à une excitation acoustique, Toulouse: ISAE, 2020.

[2] K. Kunze, C. Hirsch et T. Sattelmayer, «Transfer function measurement on a swirl stabilized premix burner in an annular combustion chamber, 》 chez ASME Turbo Expo, Vienna, 2004.

[3] L. Haber, S. W.R. et V. Khanna, «An examination of the relationship between chemiluminescent light emission and heat release rate under non-adiabatic conditions, " chez IGTI, Munich, 2000.

[4] M. Âbom et H. Bodén, "Error analysis of the two-microphone measurements in ducts with flow,» Journal of the Acoustical Siciety of America, vol. 83, pp. 2429-2438, 1988.

[5] J. M. e. a. Apeloig, "Liquid-fuel behavior in an aeronautical injector submitted to thermoacoustic instabilities,» Journal of Propulsion and Power, pp. 309-319, 2015. 